\title{
Wind climate modeling using Weibull and extreme value distribution
}

\author{
Arnab Sarkar $^{1 *}$, Sunita Singh ${ }^{2}$, Debojyoti Mitra $^{3}$ \\ ${ }^{1}$ Department of Mechanical Engineering, Institute of Technology, Banaras Hindu University, Varanasi - 221005, Uttar Pradesh, INDIA \\ ${ }^{2}$ Department of Aerospace Engineering, Indian Institute of Technology Bombay, Powai, Mumbai, Postcode 400076, Maharashtra, INDIA \\ ${ }^{3}$ Principal, Lakshmi Narain College of Technology, Indore, Madhya Pradesh, Postcode 453331, INDIA \\ "Corresponding Author: e-mail: arnab.mec@itbhu.ac.in, Tel-Mobile: (+91)8953829592
}

\begin{abstract}
It is very much important to fit wind speed data into some suitable statistical model for two aspects. One is fatigue failure due to periodic vortex shedding and the other is to estimate the wind energy potential of a particular location. For the fatigue failure due to periodic vortex shedding, it is important to analyse the load cycle. The expected number of stress cycles in the projected working life of a structure is related to the expected number of hours in the critical wind speed range and wind climate modelling is required to know this. The most popular model for this purpose is Weibull distribution. Again, wind energy is proportional to the cube of the wind speed and the same Weibull model is appropriate for cubic wind speed with different parameters. But the problem with Weibull distribution is that it fails to describe the upper tail. The aim of this paper is to determine the technique for finding the wind speed range till which Weibull model is appropriate and also to fit upper wind speed data in a suitable statistical distribution like extreme value distribution of type I (Gumbel). The hourly mean wind speed data of Ahmadabad has been used to validate the procedure.
\end{abstract}

Keywords: Wind;energy;vibration;Weibull distribution;Gumbel distribution

\section{Introduction}

Wind energy has been used for centuries for navigation and agriculture. Recently, wind energy has been receiving a lot of attention because of the focus on renewable energies. According to a report of US department of Energy (Lindenberg et al. (2008), wind energy will provide $20 \%$ electricity of US demand by 2030 . The effective utilization of wind energy entails having a detailed knowledge of the wind characteristics at the particular location. The distribution of wind speeds is important for the design of wind farms, power generators and agricultural applications like irrigation. Accurate information about wind speed is important in determining best sites for wind turbines. In recent years it has been investigated that the fitting of specific distribution to wind speed is required for use in such practical application as air pollution modeling, estimation of wind loads on building and wind power analysis (Zaharim et al., 2009). So a model is required for wind speed distribution. Phenomenon of cross wind vibration in slender structures is another aspect (Sarkar and Kasperski, 2009). Especially steel stacks are often subjected to cross wind vibrations due to Karman's mechanism. Significant cross wind motions may occur if the natural frequency of the structure is equal to the frequency of the periodic vortex shedding. The corresponding range of wind velocities is called the critical wind speed range. Periodic vortex shedding may lead to fatigue failure. The expected number of stress cycles in the projected working life of the structure is related to the expected number of hours per year in the critical wind speed range. For codification, a suitable model is required for the wind climate. The expected number of hours per year in the critical wind speed range can be determined by integrating the probability density function of hourly wind speed distribution within the critical wind speed range. Several authors (Zaharim et al., 2009; Sarkar and Kasperski, 2009; Celik, 2004; Gupta, 1986; Garcia et al., 1998; Lun and Lam, 2000; Coelingh et al., 1996; Deaves and Lines, 1997; Rehman et al. 1994; Bivona et al., 2003; Sulaiman et al., 2002) suggested Weibull model as a suitable statistical distribution for the lower wind speed range. But the upper tail cannot be fitted by Weibull model (Sarkar and Kasperski, 2009). One of the extreme value distributions is needed to fit the upper tail. In the present scope of study the statistical distributions like Weibull and extreme value distributions are discussed in section 2 . The parameter estimation and the fitting 
methodology for these distributions are presented in section 3. The results and graph for wind speed data of Ahmadabad are presented in section 4 and the final conclusion is given in section 5 .

\section{Weibull and Extreme Value Distributions}

Weibull distribution is a continuous probability distribution (named after Waloddi Weibull who described it in detail in 1951, although it was first identified by Fréchet (1927) and first applied by Rosin and Rammler (1933) to describe the size distribution of particles) which provides a close approximation to the probability laws of many natural phenomena. In recent years, most attention has been focused on this method for wind energy applications not only due to its greater flexibility and simplicity but also because of the fact that it can give a good fit to experimental data (Zaharim et al., 2009). The Weibull probability distribution function, which is a three-parameter function, can be expressed mathematically as:

$$
f(v)=\frac{k}{s-\varepsilon}\left(\frac{v-\varepsilon}{s-\varepsilon}\right)^{k-1} \exp \left[-\left(\frac{v-\varepsilon}{s-\varepsilon}\right)^{k}\right]
$$

, where $\mathrm{v}$ is the wind speed, $\mathrm{k}$ is the non-dimensional shape parameter, $\mathrm{s}$ is the scale parameter and $\varepsilon$ is the location parameter. The dimensions of $\mathrm{s}$ and $\varepsilon$ are same to $\mathrm{v}(\mathrm{m} / \mathrm{s})$. The location parameter $(\varepsilon)$ is the minimum wind speed and $\mathrm{v} \geq \varepsilon$. The cumulative distribution function can be obtained by integrating the above equation which is given below:

$$
F(v)=1-\exp \left(-\left(\frac{v-\varepsilon}{s-\varepsilon}\right)^{k}\right)
$$

Since the location parameter $(\varepsilon)$ is the minimum wind speed, it can be equated to 0 . However, by taking $\varepsilon=0$ three parameter Weibull distribution reduces to two parameter Weibull distribution. Then the probability density function and the cumulative distribution function are given by respectively:

$$
\begin{aligned}
& f(v)=\frac{k}{s}\left(\frac{v}{s}\right)^{k-1} \exp \left[-\left(\frac{v}{s}\right)^{k}\right] \\
& \text { and } F(v)=1-\exp \left(-\left(\frac{v}{s}\right)^{k}\right)
\end{aligned}
$$

The mean $(\mathrm{m})$ and variance $\left(\sigma^{2}\right)$ of the two parameter Weibull distribution are given by the following expressions:

$$
\begin{aligned}
& m_{\text {weibull }}=s \Gamma\left(1+\frac{1}{k}\right) \\
& \text { and } \sigma^{2} \text { weibull }=s^{2}\left[\Gamma\left(1+\frac{2}{k}\right)-\Gamma^{2}\left(1+\frac{1}{k}\right)\right]
\end{aligned}
$$

where $\mathrm{m}_{\text {weibull }}$ is the mean and $\sigma_{\text {weibull }}$ is the standard deviation of wind speed and $\Gamma(\mathrm{)})$ is the gamma function.

Weibull model is an appropriate model for the lower ranges of wind speed. However, for fitting purpose in the upper tail extreme value limit distributions can be used.

Extreme value theory has firstly been published in a comprehensive textbook by Gumbel (1958). He presented and discussed three basic types of extreme value limit distributions which are type I (Gumbel), type II (Fre'chet) and type III (Reverse Weibull) distributions. The probability distribution of a variate $\mathrm{x}$ can be given in the following generalised form (Kasperski, (2000):

$$
F(x)=\exp \left[-\left(f_{1}-f_{2} \cdot \frac{x-m_{\text {extreme }}}{\sigma_{\text {extreme }}}\right)^{\frac{1}{\tau}}\right]
$$

, where $\mathrm{m}_{\text {extreme }}$ is the mean value, $\sigma_{\text {extreme }}$ is the standard deviation and $\tau$ is the curvature parameter. For $\tau=0$, the extreme distribution type I (Gumbel) is obtained, i.e., in the limit equation (7) becomes equation (8):

$$
F(x)=\exp \left[-\exp \left(-\left[\gamma+\frac{\pi}{\sqrt{6}} \frac{x-m_{\text {extreme }}}{\sigma_{\text {extreme }}}\right]\right)\right]
$$

, where $\gamma$ is Euler constant $=0.5772$ 


\section{Parameter Estimation for Weibull and Gumbel Distributions}

The commonly used method for the determination of Weibull parameters are least square method, method of moment and maximum likelihood method. For this application, least square method seems to be appropriate because it gives higher weight to the lower tail. In the least square method the linearization of cumulative distribution function should be made. So from equation (4) the following relation is obtained:

$$
\ln [-\ln (1-F(v))]=k \ln v-k \ln s
$$

The equation (9) has the linear form like $y=a \cdot x+b$ where $y$ can be equated to $\ln [-\ln (1-F(v))]$ and $x$ can be equated to $\ln v$. The regression coefficients, $a$ and $b$ can be found by the method of least square. Once $a$ and $b$ are known, the shape parameter, $k$ and the scale parameter, s can be found by the following relations:

$$
\begin{aligned}
& k=a \\
& \text { and } s=e^{-\frac{b}{a}}
\end{aligned}
$$

Similarly, the parameters of type I (Gumbel) distribution can also be determined by the least square method. From equation (8) the following relation can be derived:

$$
-\ln [-\ln F(x)]=\gamma+\frac{\pi}{\sqrt{6}} \frac{x-m_{\text {extreme }}}{\sigma_{\text {extreme }}}
$$

which has the same linear form of $y=a \cdot x+b$ where $y$ can be equated to $-\ln [-\ln F(x)]$. The regression coefficients, $a$ and $b$ can be determined by the least square method. The mean $\left(\mathrm{m}_{\text {extreme }}\right)$ and the standard deviation $\left(\sigma_{\text {extreme }}\right)$ can be determined by the following equations:

$$
\begin{aligned}
& m_{\text {extreme }}=\frac{\gamma-b}{a} \\
& \text { and } \sigma_{\text {extreme }}=\frac{\pi}{\sqrt{6} a}
\end{aligned}
$$

The theoretical type I distribution is generally plotted in Gumbel probability paper where it will appear as a straight line.

\section{Results and Discussions}

The measured wind speed data in $\mathrm{km} / \mathrm{hr}$ for Ahmadabad $\left(23.03^{0} \mathrm{~N}, 72.58^{0} \mathrm{E}\right)$ is obtained from Indian Meteorological Department, Pune. The wind speed data has been measured by dyne pressure tube anemograph wind sensor (DPTA) at a height of $10 \mathrm{~m}$. The wind speed data are available in time series format where every data point represents hourly wind speed from each of 24 hours of a day. These data are sampled as 10-minute mean values taken from the last 10-minute period before the full hour. This last 10-minute mean value represents the hourly mean. All the available wind speed data have been rounded to the nearest integers. But if the decimal part of the wind speed is 0.5 , it will be rounded to the nearest odd integer. Calm hours have been eliminated from the analysis to know how much appropriate is the Weibull model to describe non-zero wind speed distribution in India. Therefore, the minimum value of the available wind speed is found to be $1 \mathrm{~km} / \mathrm{hr}$ since all wind speeds are rounded to integers. The wind speed data are supplied with class width $1 \mathrm{~km} / \mathrm{hr}$. But for getting unbiased Weibull parameters the appropriate class width has been chosen as $4 \mathrm{~km} / \mathrm{hr}$ (Sarkar and Kasperski, 2009). By converting the data into frequency distribution format with class width $4 \mathrm{~km} / \mathrm{hr}$, Weibull parameters of Ahmadabad can be estimated by least square method (Cook, 2001), as discussed in section 3. The shape parameter $(\mathrm{k})$ has been found as 1.84 and the scale parameter (s) has been found as $3.20 \mathrm{~m} / \mathrm{s}$. The histogram of probability density distribution for hourly mean wind speed data is shown below in the Figure 1. 


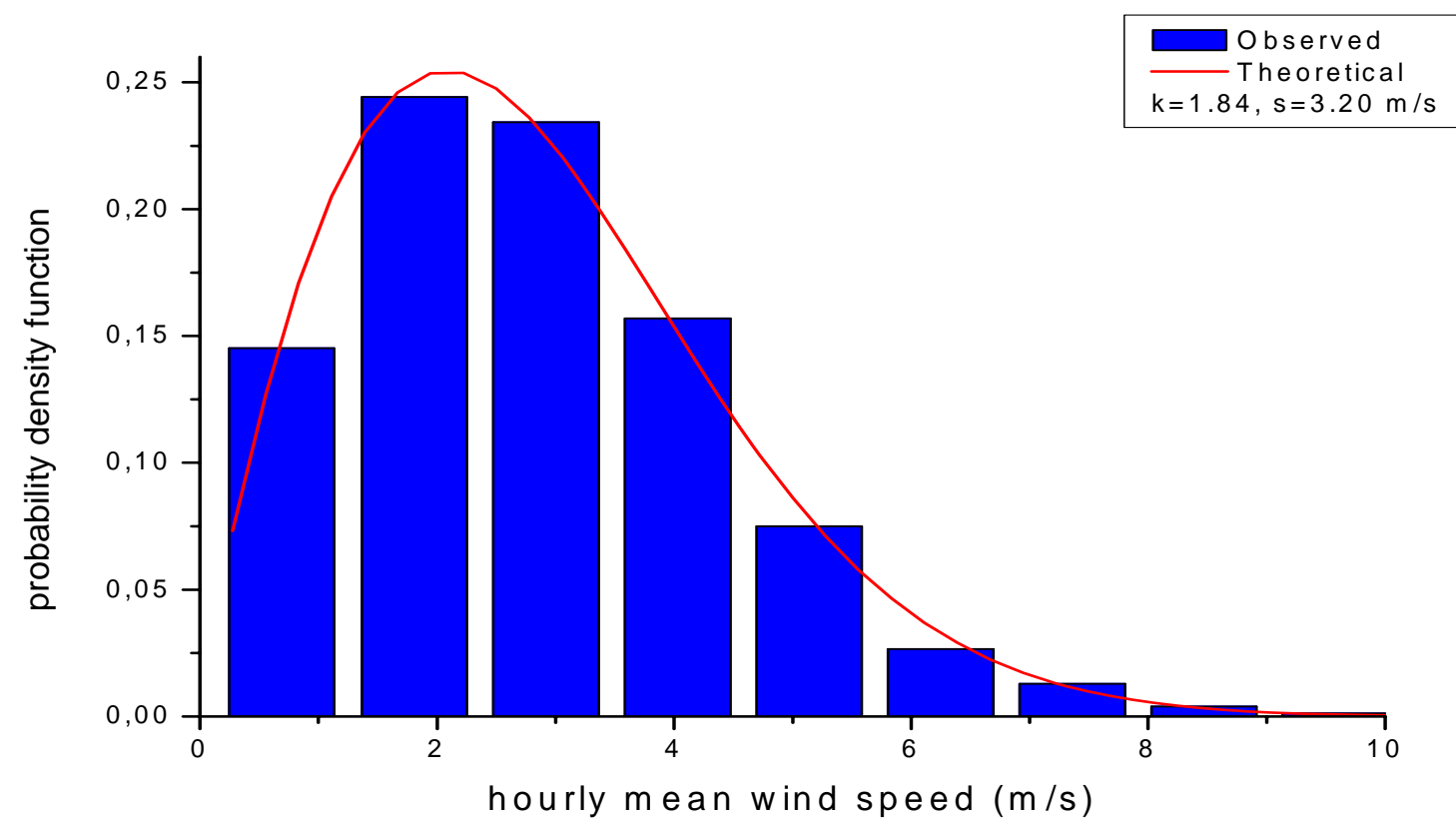

Figure 1: Probability density distribution of hourly mean wind speed data for Ahmadabad (Sarkar and Kasperski, 2009)

From the Figure 1 it is clearly understood that the theoretical model has a good agreement with the observed probability density distribution of hourly mean wind speed data. However, it is not at all clear from the figure 1 whether upper tail follows Weibull distribution or not. For this purpose $\log (1-\mathrm{F}(\mathrm{v}))$ where $\mathrm{F}(\mathrm{v})$ is the cumulative probability, is plotted with respect to hourly mean wind speed (v) in the Figure 2.

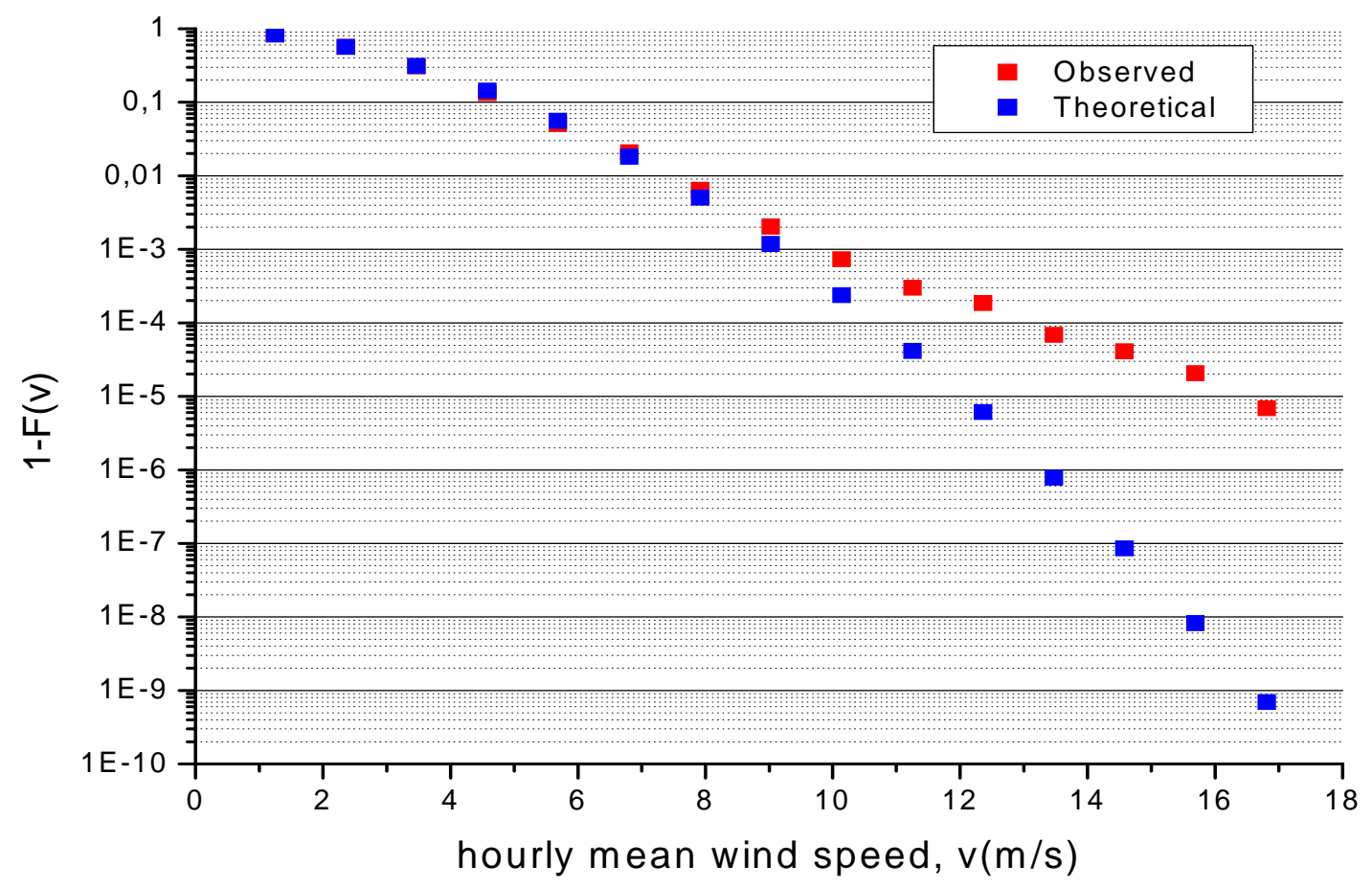

Figure 2: Probability distribution of hourly mean wind speed data for Ahmadabad (Sarkar and Kasperski, 2009) 
In the figure 2 it is clearly observed that the upper tail is not following Weibull distribution. Now it becomes necessary to find the threshold value beyond which Weibull model is inappropriate. For this purpose with shape and scale parameters $(\mathrm{k}=1.84$ and $\mathrm{s}=3.20 \mathrm{~m} / \mathrm{s}$ ) of Weibull distribution for wind speed data of Ahmadabad the mean of the distribution ( $\left.\mathrm{m}_{\text {weibull }}\right)$ can be calculated from equation (5) and it has been found as $2.843 \mathrm{~m} / \mathrm{s}$ whereas the arithmetic mean from the data has been found as $2.865 \mathrm{~m} / \mathrm{s}$. The underestimation of Weibull mean $\left(\mathrm{m}_{\text {weibull }}\right)$ is due to the fact that the upper tail of the wind speed distribution does not follow Weibull model.

Let $\lambda$ percentage of total wind speed data follows Weibull distribution and $(100-\lambda)$ percentage of total data follows extreme value distribution. So mean of the wind speed data $(\mathrm{m})$ can be expressed as follows:

$$
m=\frac{\lambda}{100} m_{\text {weibull }}+\left(1-\frac{\lambda}{100}\right) m_{\text {extreme }}
$$

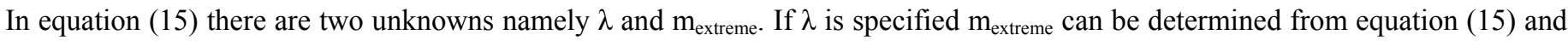
also from the wind speed data. The difference between these two values should be the least. So the value of $\lambda$ has been chosen by trial and error method in such a way so that this difference will be the minimum. In this way the value of $\lambda$ has been found as 99.64. The threshold wind speed till which Weibull distribution is appropriate and beyond which the extreme value distribution of type I (Gumbel) is appropriate, has been easily estimated from the value of $\lambda$. The threshold wind speed is found to be $8.056 \mathrm{~m} / \mathrm{s}$. Beyond this threshold value, extreme hourly mean wind speeds are sampled and their relative frequencies are calculated. Considering ensemble size sufficiently large, the relative frequency can be equated to the non-exceedance probability, $\mathrm{p}[=\mathrm{F}(\mathrm{x})]$ in the limit. The probabilities of extreme wind speeds are then fitted in type I (Gumbel) distribution. The parameters of Gumbel distribution like mean $\left(\mathrm{m}_{\text {extreme}}\right)$ and standard deviation $\left(\sigma_{\text {extreme}}\right)$ can be estimated from equations (13) and (14), as described in section 3 . In the present scope of work $\mathrm{m}_{\text {extreme }}$ and $\sigma_{\text {extreme }}$ are found to be $8.56 \mathrm{~m} / \mathrm{s}$ and $1.55 \mathrm{~m} / \mathrm{s}$. The theoretical non-exceedance probabilities can be calculated from equation (8). Both actual and theoretical probability distributions are plotted in Gumbel probability paper. In Gumbel probability paper $-\ln (-\ln p)$ vs. hourly mean wind speed are plotted for both actual and theoretical distributions. The graph is shown below in Figure 3.

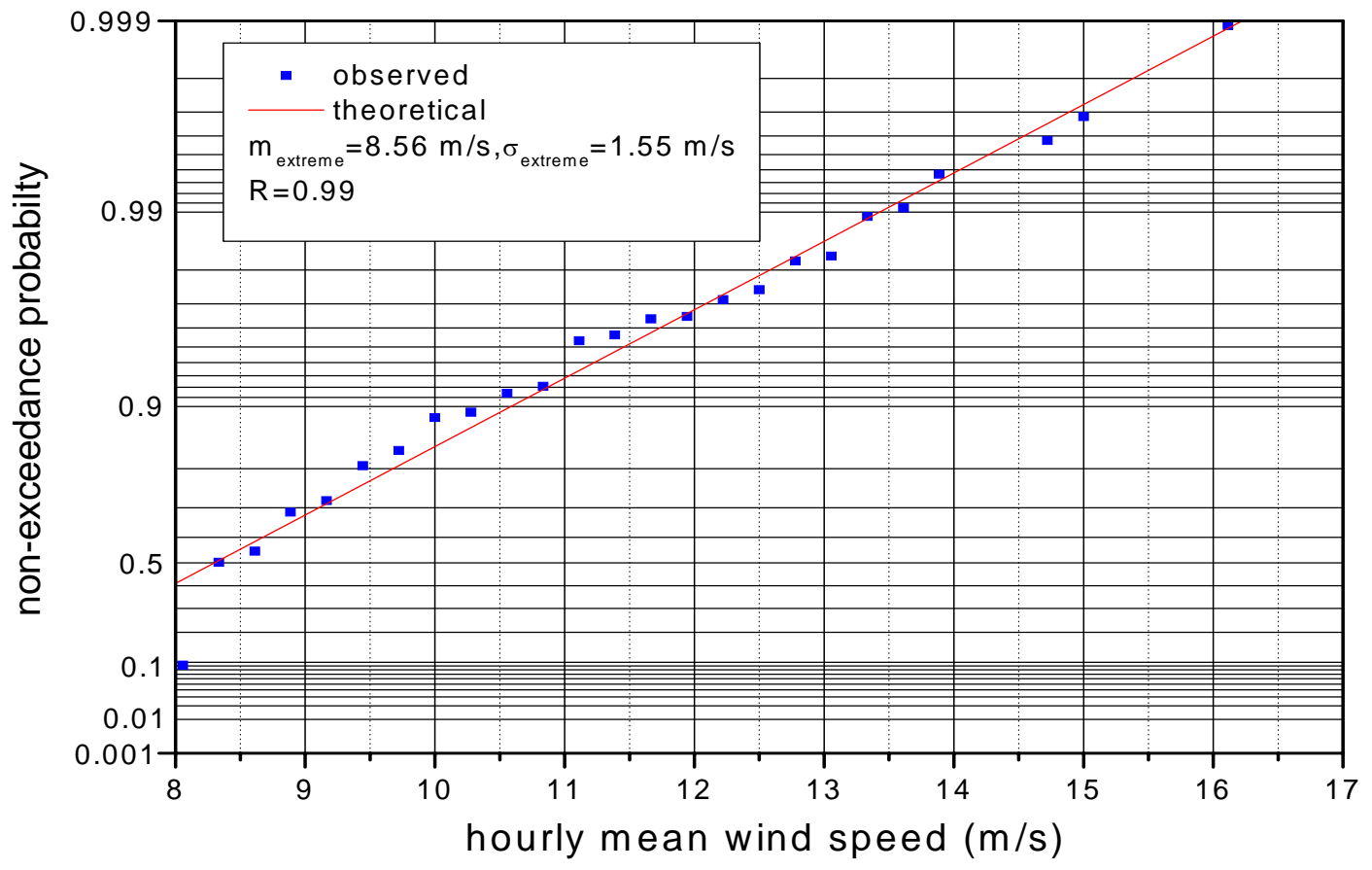

Figure 3: Non-exceedance probability of extreme hourly mean wind speed

In the Figure 3 it is interesting to note that the observed values closely follow the trend of theoretical probability. Since fitting is done only for the upper tail where Weibull model is inappropriate, $\mathrm{x}$ axis has been started from $8 \mathrm{~m} / \mathrm{s}$. The correlation coefficient (R) is found to be 0.99 which indicates that the linear fitting in the Gumbel probability paper seems to be appropriate. Now with this Gumbel distribution the upper tail in the figure 2 can be refitted which is shown below in the Figure 4. 


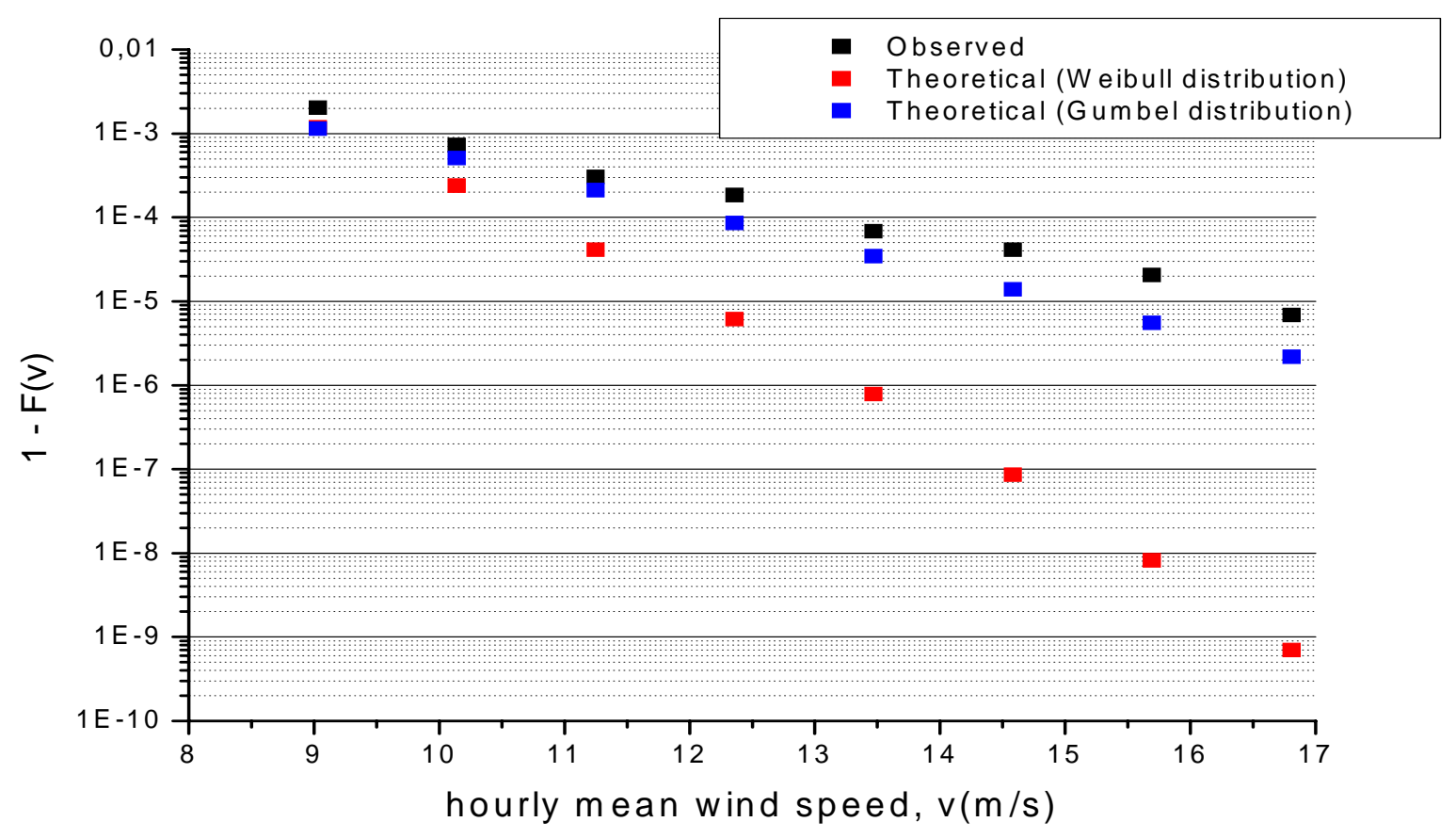

Figure 4: Probability distribution of upper hourly mean wind speed data for Ahmadabad

It is clearly observed from the figure 4 that the fitting with Gumbel model is much better than Weibull model for the upper tail. So Gumbel distribution can be suggested for modelling upper tail wind speed data.

\section{Conclusions}

Weibull model is a popular one to describe wind climate. However, there is a threshold wind speed till which Weibull model is valid and above that wind speeds can be modeled by extreme value distribution. This threshold can be analytically determined for a particular location. In case of Ahmadabad upper tail of wind speed data beyond this threshold value have been fitted by Gumbel distribution. This theoretical model has a good agreement with the observed one. In the future scope of work the upper tail can also be modeled by other extreme value distributions like type II (Fre'chet) and type III (Reverse Weibull) distributions and the best estimator can be found among these.

\section{References}

Bivona, S.; Burlon, R.; Leone, C. 2003. Hourly wind speed analysis in Sicily, Renewable Energy, Vol. 28, No. 9, pp. 1371-1385.

Fre'chet, M. 1927. Sur la loi de probabilite' de l'e'cart maximum, Annales de la Socie'te' Polonaise de Mathematique, Cracovie 6, pp. 93-116.

Celik, A.N. 2004. On the distributional parameters used in assessment of the suitability of wind speed probability density functions, Energy Conversion and Management, Vol. 45, No. 11-12, pp. 1735-1747.

Cook, N.J. 2001. Discussion on 'modern estimation of the parameters of the Weibull wind speed distribution for wind energy analysis' by J.V. Seguro, T.W. Lambert, Journal of Wind Engineering and Industrial Aerodynamics, Vol. 89, No. 10, pp. 867869.

Coelingh, J.P.; van Wijk, A.J.M.; Holtslag, A.A.M. 1996. Analysis of wind speed observations over North Sea, Journal of Wind Engineering and Industrial Aerodynamics, Vol. 61, No. 1, pp. 51-69.

Deaves, D.M.; Lines, I.G. 1997. On the fitting of low mean wind speed data to the Weibull distribution, Journal of Wind Engineering and Industrial Aerodynamics, Vol. 66, No. 3, pp. 169-178.

Gupta, B.K. 1986. Weibull parameters for annual and monthly wind speed distributions for five locations in India, Solar Energy, Vol. 37, No. 6, pp. 469-471.

Garcia, A.; Torres, J.L.; Prieto, E.; Francisco, A.de 1998. Fitting wind speed distributions: a case study, Solar Energy, Vol. 62, No. 2, pp. 139-144.

Gumbel, E.J. 1958. Statistics of Extremes, Columbia University Press, New York, London. 
Kasperski, M. 2000. Specification and Codification of Design Wind Loads, Habilitation Thesis, Ruhr-Universität Bochum, Germany.

Lindenberg, S.; Smith, B.;O’Dell, K. and et al. 2008. 20\% wind energy by 2030: increasing wind energy's contribution to U.S. electricity supply. Tech. rep., Department of Energy

Lun, I.Y.F.; Lam, J.C. 2000. A study of Weibull parameters using long term wind observations, Renewable Energy, Vol. 20, No. 2, pp. 145-153.

Rehman, S.; Halawani, T.O.; Husain, T. 1994. Weibull parameters for wind speed distribution in Saudi Arabia, Solar Energy, Vol. 53, No. 6, pp. 473-479.

Rosin, P.; Rammler, E. 1933. The laws governing the fitness of powdered coal, Journal of the Institute of Fuel, Vol. 7, pp. 29-36.

Sarkar, A.; Kasperski, M. 2009. Weibull parameters for wind speed distribution in India, Proceedings of $5^{\text {th }}$ National Conference on Wind Engineering, pp. 134-158.

Sulaiman, M.Y.; Akaak, A.M.; Wahab, M.A.; Zakaria, A.; Sulaiman, Z.A.; Suraidi, J. 2002. Wind characteristic of Oman, Energy, Vol. 27, No. 1, pp. 35-46.

Zaharim, A.; Razali, A.M.; Abidin, R.Z.; Sopian, K. 2009.: Fitting of statistical distributions to wind speed data in Malaysia, European Journal of Scientific Research, Vol. 26, No. 1, pp. 6-12.

\section{Biographical notes}

Arnab Sarkar is an Assistant Professor in the Department of Mechanical Engineering, Institute of Technology, Banaras Hindu University, Varanasi, India. He received his Master of Mechanical Engineering from Jadavpur University, Kolkata, India in 2003. He is currently pursuing his Ph.D. in the same university. He was a DAAD Doctoral Fellow in Ruhr-University Bochum, Germany from October, 2007 to September, 2009. His research interests include fluid dynamics, wind engineering, building aerodynamics and wind energy. He presented almost ten research articles in national and international conferences and received national and international awards for his academic performances.

Sunita Singh is a fourth year integrated dual degree student in the department of Aerospace Engineering in Indian Institute of Technology Bombay, India. Her research interests include propulsion, fluid dynamics and building aerodynamics.

Dr. Debojyoti Mitra is currently working as the Principal of Lakshmi Narain College of Technology, Indore, India. He was former Associate Professor and Head in the department of Mechanical Engineering of Sir Padampat Singhania University, Udaipur, India and was also Senior Lecturer of Jadavpur University. He has more than 13 years of experience in teaching and research. He also has post doctoral research experience in Germany and Canada. His current area of research includes Wind Engineering, Industrial Aerodynamics, Environment and Pollution. He has published about 15 papers in referred international journals and presented about 30 research articles in national and international conferences. He is also the member of editorial boards of many journals.

Received March 2011

Accepted March 2011

Final acceptance in revised form May 2011 